
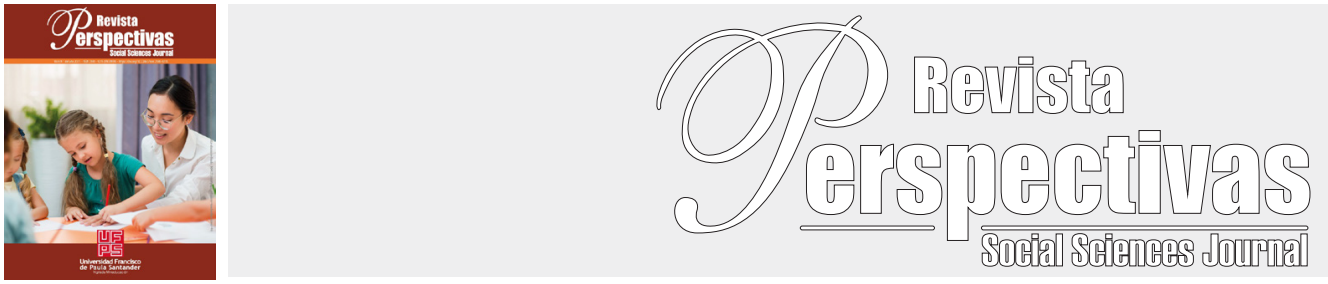

Artículo Original

https://doi.org/10.22463/25909215.2924

\title{
Estudio de las dinámicas urbanas del sector de cabecera del llano como herramienta pedagógica para la arquitectura
}

\section{Study of the urban dynamics of the cabecera de llano's sector as a pedagogical tool for architecture}

Fernando Andrés Estévez-Suarez ${ }^{1}$, Luz Karime Coronel-Ruiz², Fabio Andrés Lizcano-Prada ${ }^{3}$

'Arquitecto, Magister en Ordenamiento Territorial, Universidad Santo Tomás Bucaramanga, Colombia, Grupo de Investigación GINVEARQUI, fernando.estevez@ustabuca.edu.co, ORCID:0000-0002-9512-625X 'Arquitecta, Magister en Gestión Urbana, Universidad Francisco de Paula Santander, Cúcuta, Colombia, Grupo de Investigación TARGET, luzkarimecr@ufps.edu.co, ORCID:0000-0003-033I-0568

${ }^{3}$ Arquitecto, Magister en Ordenamiento Territorial, Universidad Santo Tomás Bucaramanga, Colombia, Grupo de Investigación GINVEARQUI, fabio.lizcano@ustabuca.edu.co, ORCID:0000-0002-1336-6509

Cómo citar: F.A. Estévez-Suarez, L.K. Coronel-Ruiz, F.A. Lizcano-Prada "Estudio de las dinámicas urbanas del sector de cabecera del llano como herramienta pedagógica para la arquitectura". Perspectivas, vol. 6, no. 1, 59-71. 2021

Recibido:Agosto 15, 2020; Aprobado: Noviembre 23, 2020.

\begin{tabular}{ll}
\hline Ralabras claves: & RESMEN \\
\hline urbana; y, como herramienta pedagógica para el área de la arquitectura. En el se abordan las transformaciones y \\
Análisis urbano, & tendencias de uso del suelo que se han desarrollado en el sector de Cabecera del Llano, Santander - Colombia \\
Ciudad, & entre los años 2008-2019 desde un enfoque histórico, territorial y pedagógico, con relación a los componentes \\
Gestión Territorial, & físico-construido, urbano-ambiental, social-cultural y económico- normativo; con el fin de proponer aspectos \\
Transformaciones urbanas. & significativos o de interés para la solución de problemáticas presentes en el territorio, y plantear orientaciones \\
& como respuesta a las tendencias o transformaciones del uso del suelo. El marco metodológico de esta \\
& investigación se enmarca en el paradigma mixto, es de tipo descriptivo, y esta basado en el desarrollo de un \\
& estudio de campo que responde a lo transversal, observacional y retrospectivo, que soporta y complementa \\
& el componente teórico planteado dentro de la misma. Las herramientas de investigación utilizadas dentro de \\
& esta metodología corresponden a la observación participante, los diarios de campo, el análisis urbano y una \\
& matriz de resultados. Como hallazgo relevante se destaca que en razón de los cambios significativos en el \\
ordenamiento territorial y en las tendencias de uso del suelo evidenciadas en Cabecera del Llano para el periodo & de estudio, es necesario fortalecer el control urbano en referencia a los usos comercial y dotacional, con el fin \\
de que estos den cumplimiento a lo establecido en la guía territorial, así como generar estudios proyectivos \\
constantes con relación a los cambios del uso del suelo, teniendo en cuenta que las dinámicas allí presentes son \\
altamente variables.
\end{tabular}

\section{ABSTRACT}

\section{Keywords:}

Urban analysis,

City,

Territorial Management,

Urban transformations.
This research article highlights the importance of territorial analysis within urban management; and, as a pedagogical tool for the area of architecture. It addresses the transformations and trends in land use that have developed in the sector of Cabecera del Llano, Santander - Colombia between 2008-2019 from a historical, territorial and pedagogical approach, in relation to the physical-built components, urban-environmental, social-cultural and economic-normative; in order to propose significant or interesting aspects for the solution of problems present in the territory, and propose guidelines in response to trends or transformations in land use. The methodological framework of this research is framed in the mixed paradigm, it is descriptive, and is based on the development of a field study that responds to the cross-sectional, observational and retrospective aspects, which supports and complements the theoretical component raised within the herself. The research tools used within this methodology correspond to participant observation, field diaries, urban analysis, and a results matrix. As a relevant finding, it is highlighted that due to the significant changes in land use planning and trends in land use evidenced in Cabecera del Llano for the study period, it is necessary to strengthen urban control in reference to commercial and endowment uses, In order for them to comply with the provisions of the territorial guide, as well as generate constant projective studies in relation to changes in land use, taking into account that the dynamics present there are highly variable. 


\section{Introducción.}

El presente artículo se deriva de la investigación denominada "TRANSFORMACIÓN DEL TERRITORIO POR DINAMICAS URBANAS. COMPARATIVOENTREDOSSECTORESDELAS CIUDADES DE CÚCUTA Y BUCARAMANGA" realizada bajo la alianza interinstitucional a través de los grupos de investigación GINVEARQUI (Universidad Santo Tomas) y TARGET (Universidad Francisco de Paula Santander). Se centra en la reflexión sobre los procesos de expansión urbana que se desarrollan dentro de las ciudades y que transforman sus proyecciones iniciales de acuerdo a la demanda de servicios y cambios de uso del suelo en respuesta a su crecimiento físico-construido, teniendo en cuenta lo establecido por CEPAL (2013) en la Guía análisis del sistema urbano regional para el ordenamiento territorial, División de Desarrollo Sostenible y Asentamientos Humanos.

El área o polígono de estudio se ubica en la comuna 12 de la ciudad de Bucaramanga, departamento de Santander, Colombia, esta comprendido por las calles 41 y 42 en sentido norte, calle 56 sentido sur con la diagonal, por el oriente con la avenida el jardín (avenida 42 y la escarpa) y por el occidente con la carrera 33. El polígono está conformado por 78 manzanas, este barrio cuenta con diferentes ejes vehiculares de acceso. Con respecto al uso del suelo, se destaca que las calles 56 y 45 y las carreras 33 y 36 , presentan uso residencial bajo una tipología de vivienda unifamiliar y multifamiliar, además un uso comercial, en el que se destacan la zona denominada como cabecera 1, 2, 3 etapa y los centros comerciales Cuarta y Quinta Etapa. En lo referente a servicios dotacionales, el barrio que cuenta con la unidad Clínica Bucaramanga, y otros servicios de salud de atención médica, además de infraestructura o equipamiento educacional, y cuenta con espacios de esparcimiento público como el parque San Pio.

El barrio Cabecera del Llano se presenta como una centralidad, que juega un papel fundamental no solo a nivel municipal, sino metropolitano, que da cumplimiento a lo establecido por teóricos como Karl Brunner en Beuf (2012), para quien las nuevas centralidades deben acompañar los procesos de crecimiento y desarrollo urbano, otorgando nuevos equipamientos y variedad de usos que fomenten la identidad territorial y el equilibrio en el territorio. En este sentido, es importante recordar que el barrio Cabecera del Llano fue proyectado en los años 50 y a lo largo de su historia ha atravesado diferentes procesos de transformación a nivel urbano, asociados al fenómeno de crecimiento o expansión de la ciudad.

En este proceso de investigación se desarrolló dentro de un marco histórico que comprenden los años 1998 y 2019, y realizó un análisis basado en las tendencias del uso del suelo relacionadas al aumento notable del valor del metro cuadrado, la inseguridad, factores de orden cultural y social; y aspectos relacionados al carácter ambiental y económico, con el fin de establecer estrategias que puedan ser implementadas dentro de las practicas pedagógicas y las investigaciones del área de la arquitectura, así como para la generación de lineamientos que puedan contribuir al ordenamiento territorial de la ciudad.

Consecuentemente, esta investigación presenta un análisis urbano realizado para el polígono de estudio, en el cual se identifican los principales aspectos de interés para los componentes: físico, medioambiental, social, cultural, económico $\mathrm{y}$ gubernamental, desarrollados durante el periodo de tiempo objeto de estudio. Finalmente, se presentan aspectos de interés para la gestión urbana y la practica pedagógica de arquitectura, consolidados a partir de la elaboración de una matriz que permite el análisis y la formulación de estrategias de intervención urbana.

\section{Material y método.}

El marco metodológico de esta investigación se enmarca en el paradigma mixto y es de tipo descriptivo. El diseño se centra en un estudio de campo que responde a lo transversal, observacional y retrospectivo, que como objetivo ser el soporte y 
complemento del marco teórico desarrollado dentro de la misma.

Desde esta perspectiva, la metodología de investigación mixta planteada para esta propuesta se apoya en el modelo metodológico racionalista cuantitativo, teniendo en cuenta que a través de este se obtienen datos tangibles, rigurosos y fidedignos; adquiridos a partir de procedimientos de fácil revisión y de carácter sistemático (Bryman, 1998). Este hecho, trae como consecuencia la generación de un proceso de análisis concreto, desarrollado a partir de la interrelación de variables que favorecen la identificación de patrones generales (Skinner, Hollovay, \& Tagg, 2000). Por otro lado, se fundamenta en el modelo naturalista cualitativo, basado en actividades exploratorias a través de las cuales se pueden captar datos, experiencias, percepciones y hechos in situ correspondientes a la realidad del área sometida a estudio (Vélez de la Calle, 1999), (Gill \& Johnson, 2010).

La utilización de este marco metodológico favorece la flexibilización del uso de las herramientas de recolección de datos que permiten obtener una información más detallada, que posteriormente puede ser triangulada (Molina Azorín, 2012) así como la generación de una visión holística en relación con el reconocimiento de las dinámicas y componentes urbanos generadores de la transformación territorial en el área sometida a estudio.

Como herramientas metodológicas se estableció la Observación participante, entendida como la técnica cualitativa más empleada para analizar el componente socio-cultural, a partir de la inmersión en la realidad propia del territorio (Guash, 1997). Así mismo, el diario de campo como un instrumento de registro de información procesal, organizado metódicamente con respecto a la información que se desea obtener en cada uno de los reportes, a partir de diferentes técnicas de recolección de información que permiten la identificación de la realidad del área de estudio (Martínez R, 2007). Consecuentemente, se implementó el análisis del contexto que hace referencia a una metodología que vincula la teoría y la práctica, por medio de la integración con las ciencias humanas, a través de disciplinas como la sociología, la antropología y la sociología, estableciendo relaciones más directas entre el proyecto el habitante el contexto y la ciudad (Gallardo Frías, 2015) y finalmente, la matriz de resultados prevista como una herramienta de análisis, comprensión y generación de estrategias que favorece la practica pedagógica, en razón al desarrollo de procesos que incluyen relaciones causales entre la formulación de acciones, ejecución de resultados y propuesta de productos e insumos (propósitos, aspectos significativos o claves y orientación para el estudio o análisis en el sector de estudio). (Aldunate, 2004; Banco Interamericano de Desarrollo- BID, 1997; World Bank, S.F)

Dentro de las fases previstas dentro de este marco metodológico se encuentran:

Fase 1: Estudio cronológico en periodo de tiempo 1998-2019. Esto con el fin de obtener información sobre la creación de tendencias de ocupación del suelo urbano, con el apoyo de documentos de gestión territorial, el desarrollo de salidas de campo, información de indicadores poblacionales, planos, mapas del polígono de estudio, apoyados en la guía planteada por Bernal Granados (2015).

Fase 2: Diagnóstico de la situación actual del polígono de estudio, de acuerdo con el análisis entre los efectos de los atributos urbanos y las actuales tendencias de ocupación del suelo, basado en conceptos como: compacidad, la complejidad y la eficiencia (Agencia de Ecología Urbana de Barcelona, 2008).

Fase 3: Generación de una matriz de reconocimiento de cambios en los usos del suelo para evidenciar las tendencias de uso del suelo en el sector de Cabecera del Llano en Bucaramanga, proponer estrategias para el fortalecimiento de la guía territorial y la practica pedagógica en la Arquitectura referente a temas de ordenamiento y gestión territorial. 


\section{Resultados y discusión.}

El proceso de los resultados se lleva acabo a partir de la revisión de los hechos históricos, el marco teórico y el trabajo de campo realizado para esta investigación. El cual fue analizado dentro del marco temporal establecido (21 años), y centrado en un proceso descriptivo. Lo anterior, se presenta como una herramienta pedagógica que permite profundizar y reconocer aspectos relevantes dentro del ordenamiento y la gestión territorial, por medio de los cuales se pueden obtener datos significativos para el entendimiento de las transformaciones a nivel urbano y las tendencias de ocupación en los usos del suelo.

La ciudad de Bucaramanga es la capital del departamento de Santander, se encuentra ubicada sobre la cordillera oriental al nororiente del país, tiene una población de 1.111 .999 habitantes incluyendo su área metropolitana, según datos del DANE (2018). Su territorio se encuentra comprendido por 17 comunas, dentro de las cuales hacen parte los barrios, asentamientos, urbanizaciones y otros (sectores con población flotante), según el plano UR1 denominado División Urbana, tomado del Sistema de información georreferenciado del Municipio de Bucaramanga (Figura 1).

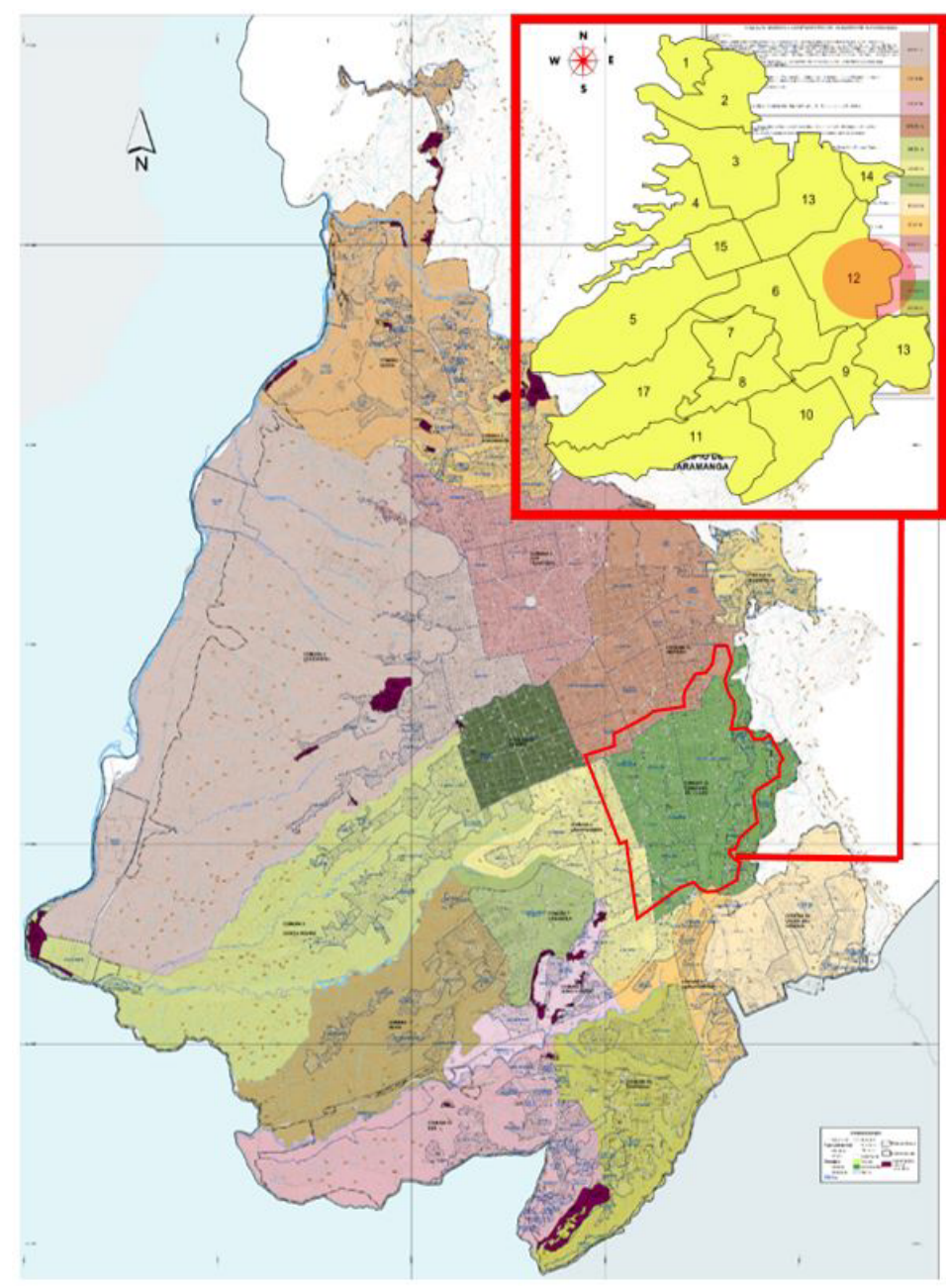

Figura 1. Plano de división Político Urbana de Bucaramanga

Fuente: Tomado de: https:/www.bucaramanga.gov.co/el-mapa/division-politico-urbana/ 
Autores como Vera Rojas (2017) mencionan que el barrio Cabecera del Llano inició su desarrollo urbano a partir de los años 50, sobre un terreno de $676.696 \mathrm{~m} 2$ visionado como un punto estratégico para Bucaramanga, siendo la constructora Puyana la encargada de la venta de los lotes de vivienda unifamiliar de dos plantas dirigida al estrato 6 , y diseñadas con un sistema constructivo tradicional; considerando además dentro del barrio la implementación de otros usos como: comercio, servicios complementarios y recreación.

Tabla 1. Áreas de tipo residencial y comercial en el barrio Cabecera del llano

\begin{tabular}{|l|c|c|l|}
\hline \multicolumn{4}{|c|}{ Sector Cabecera del Llano } \\
\hline \multirow{3}{*}{ Terreno } & Área Total M $^{2}$ & 676.696 & $100 \%$ \\
\cline { 2 - 4 } & Área Residencial M & 341.886 & $51 \%$ \\
\cline { 2 - 4 } & Área Comercial M $^{2}$ & 333.810 & $49 \%$ \\
\hline \multirow{3}{*}{ Construido } & Área Total M $^{2}$ & 999.362 & $100 \%$ \\
\cline { 2 - 4 } & Área Residencial M & \\
\cline { 2 - 4 } & Área Comercial M & $77 \%$ \\
\hline
\end{tabular}

Fuente: Ficha predial Agustín Codazzi, consultado en 2019

Asimismo, el autor establece que en los años 70 la constructora Urbanas desarrolló de manera exitosa la etapa I del complejo comercial y dio paso a la construcción de la II, III, IV y V etapa; consecutivamente, promoviendo la generación de nuevos usos del suelo asociados al sector salud, comercio, hotelería, entretenimiento y otros. Con respecto al espacio público, se destaca la construcción del parque San Pio, catalogado como un hito urbano de carácter metropolitano y de gran reconocimiento por parte de la comunidad. Dentro del barrio también se encuentran ubicados los equipamientos educativos: la Presentación, la Merced y la Institución de Educación Superior Universidad Autónoma de Bucaramanga.

Dentro del proceso de transformación urbana, se resalta que en los años 80 se generó un cambio de tipología de vivienda unifamiliar a vivienda multifamiliar en altura (de más de 10 pisos), así como el cambio de uso residencial a comercial (Rueda Gómez, 2012). Es importante tener en cuenta que sobre la carrera 33 se gestó y desarrolló un plan de recuperación de espacio público, que constaba de la ampliación y terraceo de las circulaciones peatonales, adecuando el parque San Pio para generar un parqueadero debajo de las canchas deportivas, en respuesta a las necesidad que presentaba el sector.

Es necesario mencionar que a la fecha aún se presentan procesos de transformación asociados a las actividades comercial, dotacional, residencial y de actividad múltiple, hecho que ha dinamizado el sector y su importancia para la ciudad de Bucaramanga. Lo anterior cobra relevancia al tener en cuenta posturas de autores como Contreras (2009) quien establece la importancia de comprender las transformaciones del territorio a partir de cada uno de sus componentes. Así las cosas, para este autor los cambios asociados al uso residencial (recuperación, renovación y movilidad) están relacionados con los cambios de la estructura productiva del territorio y las dinámicas socio-culturales de la población que lo habita.

Según el Plan de Ordenamiento Territorial de segunda generación POT (2014-2027) el barrio Cabecera del llano presenta áreas de actividad residencial $\mathrm{T} 1, \mathrm{~T} 2$, con viviendas unifamiliares y multifamiliares de estratos 5 y 6 , áreas de actividad Dotacional T1, áreas de actividad Múltiple T1, donde se destacan edificaciones de oficinas privadas, zonas de recreación como el parque San Pio con zonas verdes y deportivas, áreas de actividad Comercial $\mathrm{T} 1$, donde se destaca el comercio como las etapas I.II,III, y los centros comerciales IV y V etapa, este polígono cuenta con cuatro vías importantes las carreras 33 y 36 y las calles 45 y 56 (Tabla 2). 
Tabla 2. Clasificación zonal del barrio 1975-2019

\begin{tabular}{|c|c|}
\hline Año & $\begin{array}{c}\text { Zonificación del Barrio Cabecera del llano a } \\
\text { través de los años }\end{array}$ \\
\hline 1945 & Zona residencial nueva según el código de Urbano \\
\hline 1982 & $\begin{array}{c}\text { Zona desarrollada. } \\
\text { Zona de transformación o renovación indirecta }\end{array}$ \\
\hline 1984 & $\begin{array}{c}\text { Zona residencial } \\
\text { Zona Mixta }\end{array}$ \\
\hline 2000 & $\begin{array}{c}\text { Zona de actividad Comercial T1 } \\
\text { Zona de actividad Dotacional T1 } \\
\text { Zona de actividad Múltiple T1 } \\
\end{array}$ \\
\hline $2014-$ & $\begin{array}{c}\text { Zona de actividad Residencial T1, T2, T3 } \\
\text { Zona de actividad residencial T1, T2 Dotacional T1 } \\
\text { Zona de actividad Múltiple T1 } \\
\text { Zona de actividad Comercial T1 }\end{array}$ \\
\hline
\end{tabular}

Fuente: Elaboración propia a partir del Plan de Ordenamiento Territorial de segunda generación 2014-2027 Acuerdo 011.

En el año 2019 en el barrio Cabecera del Llano predominó el uso comercial con un $59 \%$, el $2.99 \%$ correspondió al uso dotacional, el $5.88 \%$ a residencial- comercio, el $30 \%$ correspondió a vivienda unifamiliar y multifamiliar y el $1 \%$ a predios si edificación. Se destaca que a partir del análisis urbano realizado para esta investigación se pudo establecer que, dentro del proceso de desarrollo urbano, el uso comercial ha sido uno de los principales detonantes de transformación urbana, debido al cambio de tipología de uso del suelo presente a lo largo de la historia del sector objeto de estudio (Figura 2).

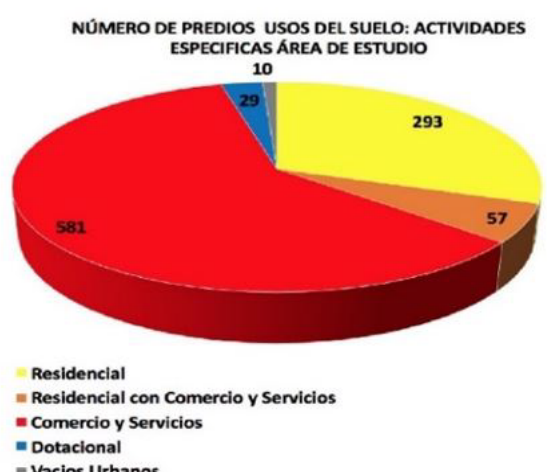

- Dotacional

PORCENTANE USOS DEL SUELO: ACTIVIDADES ESPECIFICAS ÁREA DE ESTUDIO

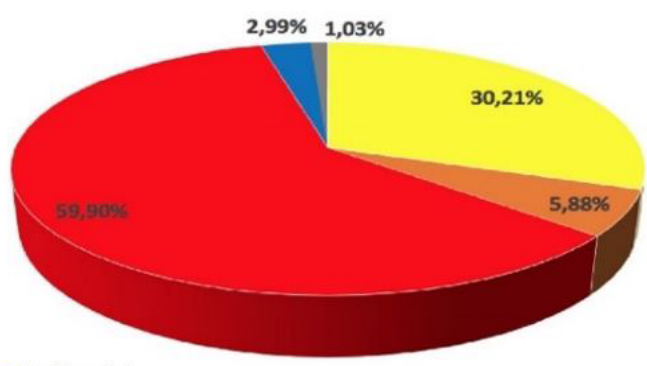

Residencial

w Residencial con Comercio y Servicios

- Comercio y Servicios

- Dotacional

Eacios Urbanos

Figura 2. Número de predios y porcentajes de usos del suelo 2019.

Fuente Elaboración propia

Igualmente, la infraestructura del sector también hace parte de las transformaciones de las tendencias del uso del suelo, al tener en cuenta que el barrio cuenta con el desarrollo de centros de atención de salud como la Clínica Bucaramanga, así como consultorios médicos y odontológicos, además se resalta para este sector el tratamiento y el mejoramiento del espacio público como elemento integrador a nivel urbano en la zona, permitiendo reivindicar la importancia del peatón, los espacios verdes y los recreo-deportivos que propician el bienestar de la comunidad. Lo anterior puede ser ejemplificado a través del proyecto de recuperación de espacio público realizado por la administración en la carrera 33.

Descripción del Polígono de Estudio: El polígono de estudio está comprendido entre las carreras 33 y 38 y las calles 42 y 56 de la ciudad de Bucaramanga, en el se concentra el uso residencial a partir de la vivienda unifamiliar y multifamiliar, 
asimismo se destaca el alto porcentaje de predios destinados al comercio, y los cambios de uso del suelo que se han generado en el proceso de crecimiento urbano durante los últimos veintiún años. Además, el polígono de estudio cuenta con servicios educativos, de salud y una infraestructura vial que soporta el sector.

El proceso de crecimiento urbano del sector objeto de estudio, evidencia que para el año 1939 se generó el nacimiento del barrio cabecera del Llano en los predios de David Puyana, específicamente en la hacienda "Valle de don Andrés". Entre los años de 1938-1952 se inició la construcción de varias casas quintas que ocuparon el costado occidental, iniciando un proceso de desarrollo urbano importante en el municipio de Bucaramanga. Entre 1960-1970 se aprobó un loteo completo del Valle de don Andrés, con el fin de desarrollar alternativas de vivienda, y se proyectó la construcción del Centro Comercial Primera Etapa, considerado como el detonante del uso comercial en el polígono de estudio. Asimismo, la construcción de viviendas unifamiliares en el sector entre los años 1970-1990 generó un incremento en los indicadores sociales, que aumentó el número hogares residentes en el sector (Estévez Suarez, 2018).

Consecuentemente, se construyen las etapas 2, 3, 4 y 5 de los centros comerciales, y entre los años 1990 -2018 se presentó un incremento en el valor del suelo por metro cuadrado, hecho que generó un aumento en el índice de ocupación motivado a la construcción en altura, determinando así transformaciones en el paisaje urbano, afectaciones de tipo ambiental y el traslado del uso del suelo residencial a comercial (Gomez, 2003; Vera, 2018).

En la siguiente figura se puede observar como el código de edificaciones de Bucaramanga (1940) evidencia que la ciudad de Bucaramanga estaba conformada por cinco zonas: centro, norte, oriental, concordia y vivienda obrera, mientras que en el código urbano de 1945 se establecen las zonas de acuerdo al tipo: comercial, residencial antigua, residencial nueva, industrial, mixta y suburbana. Finalmente, para 1982 el código de urbanismo señala un cambio en la estructura basado en la zonificación contemplando las zonas: comercial tipo 1, dotacional tipo 1, múltiple tipo 1, residencial tipo 1 y 2 y zonas verdes.
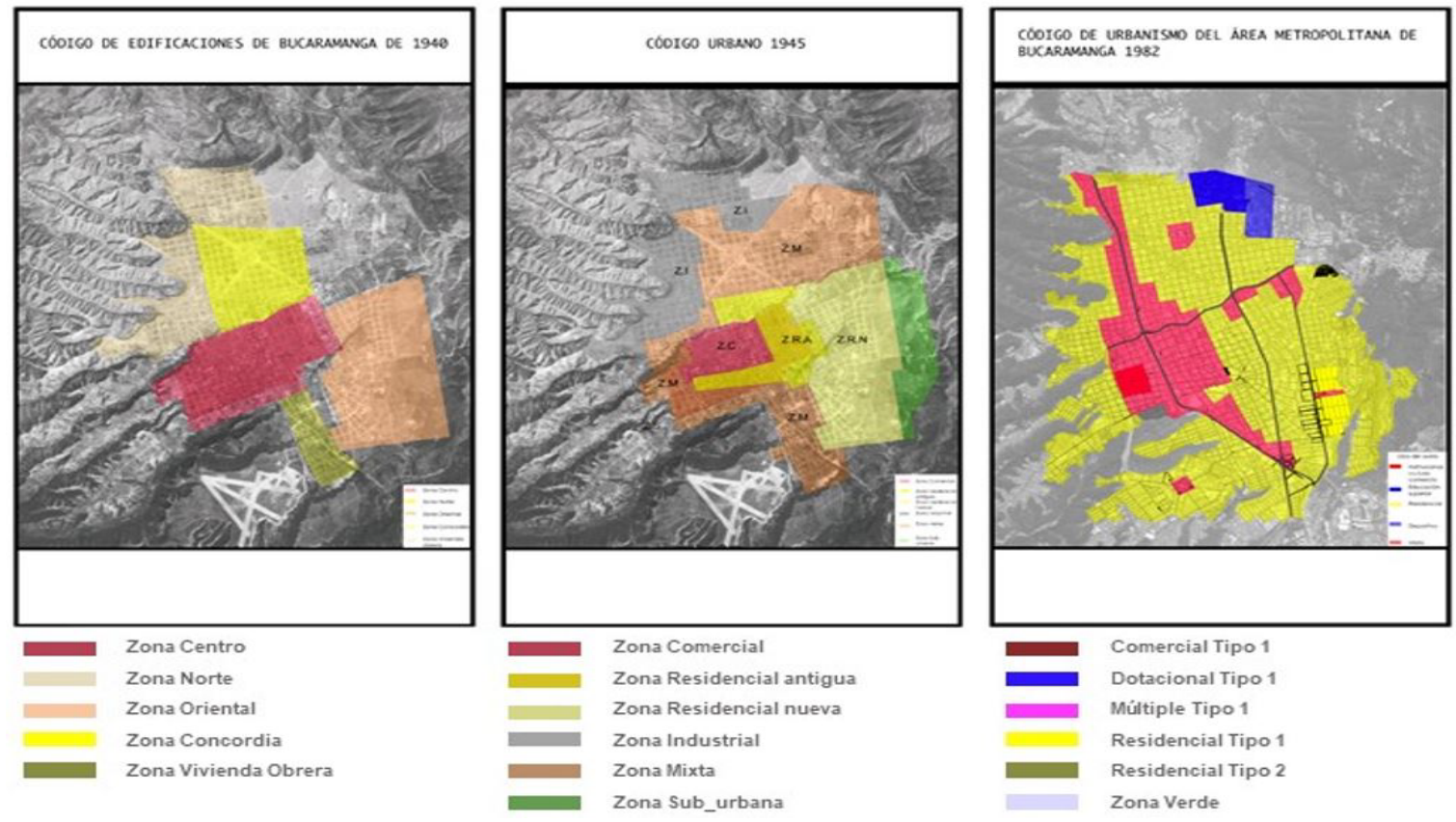

Figura 3: Crecimiento urbano Bucaramanga 1940-1982

Fuente:Elaboradas a partir de el código de urbanismo (1940), código urbano (1945), código de urbanismo del Área Metropolitana (1982) 
A partir del año 2000 en Bucaramanga adopto el Plan de Ordenamiento Territorial- POT, en el cual se observan los usos de tipo comercial tipo 1, dotacional tipo 1, múltiple tipo 1, residencial tipo 1, residencial tipo 2, residencial tipo 3 y zonas verdes. Posteriormente, en el 2014 se aprobo el Plan de Ordenamiento de Segunda Generación 2014-2027, en el cual se contemplan los siguientes usos: comercial tipo 1, dotacional tipo 1, múltiple tipo 1, residencial tipo 1, residencial tipo 2 y zonas verdes, asi las cosas, en Cabecera del Llano se establece un nueva centralidad en la que se pueden apreciar los usos: residencial, residencial con comercio y servicios, comercio y vivienda dotacional y predios no construidos: Lo anterior permite apreciar el cambio de tendencias del suelo por medio de la aparición de usos mixtos que actualmente no cumplen con lo establecido por la guia territorial vigente (Concejo de Bucaramanga, 2014; Gomez, 2003; Vera, 2018).
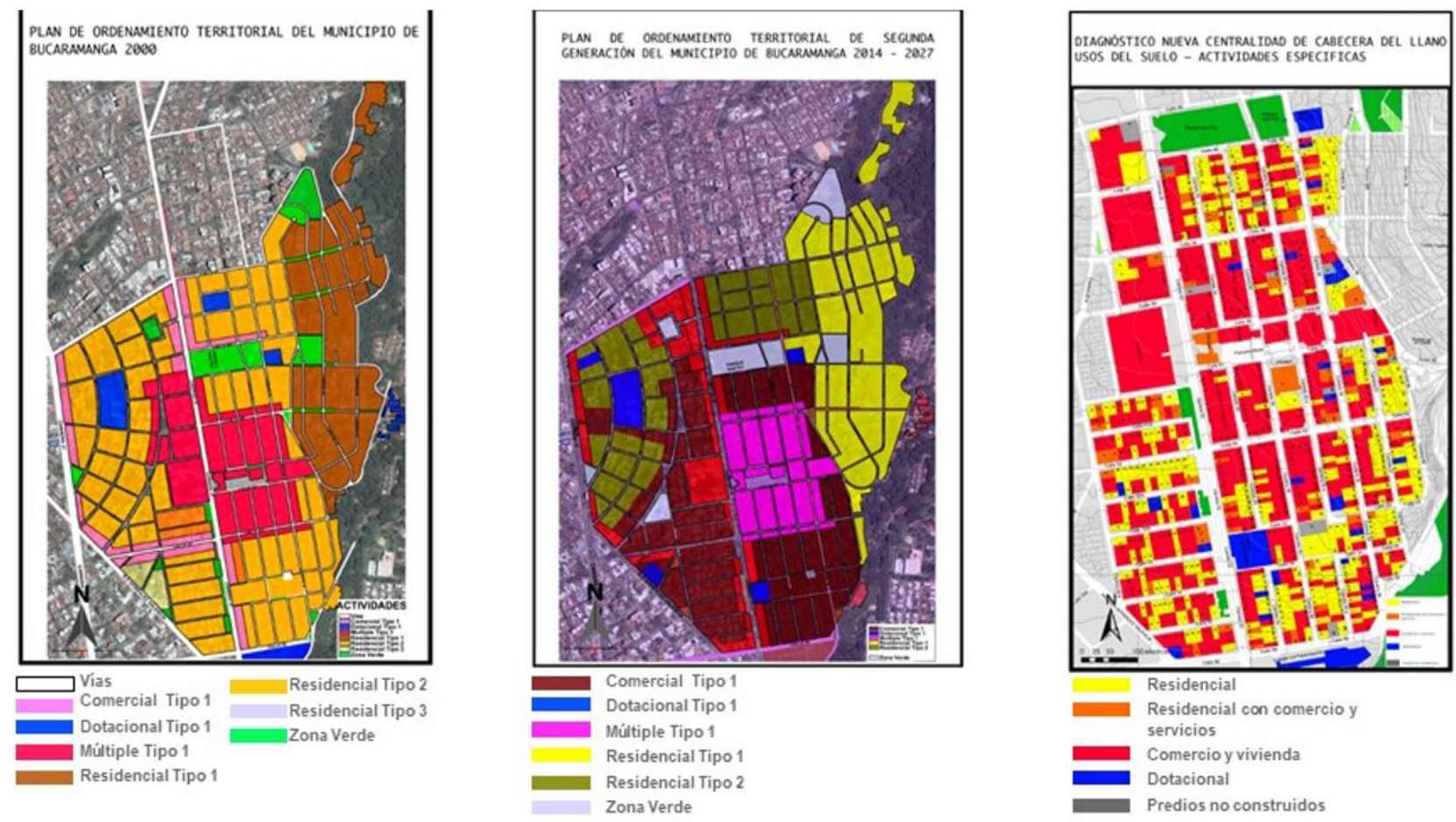

Figura 4: Imágenes de los instrumentos de gestión

Fuente: Elaboración propia a partir de Plan de Ordenamiento Territorial Bucaramanga (2000) Plan de Ordenamiento Territorial segunda generación (2012-2027) y Diagnostico nueva centralidad de Cabecera del Llano.

En el último decenio, se pudo observar que el uso comercial presentó la mayor fuerza de ocupación del sector objeto de estudio, lo que motivo el aumento de los costos de arriendo a raíz de la consolidación de las plataformas comerciales. Asimismo, el uso residencial perdió fuerza y se presentó una transformación tipológica residencial en donde la vivienda unifamiliar da paso a la multifamiliar en altura. Con respecto al uso dotacional, se considera que a la fecha el sector objeto de estudio da cumplimiento básico a las necesidades de la población residente. 


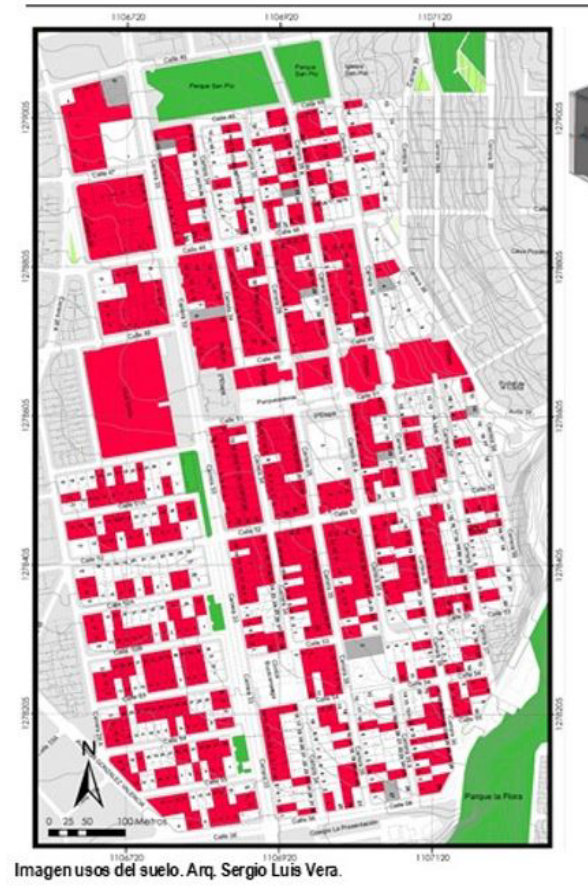

Imagenusos del suelo. Arq. Sergio Luis Vera.

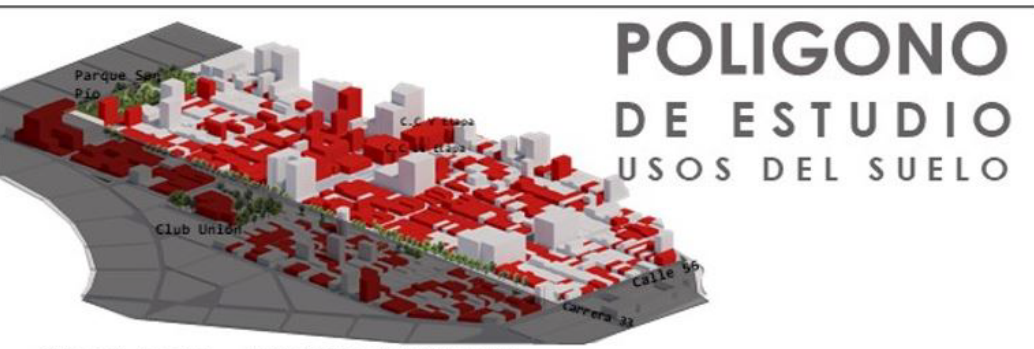

USO DEL SUELO - COMERCIAL Y SERVICIOS

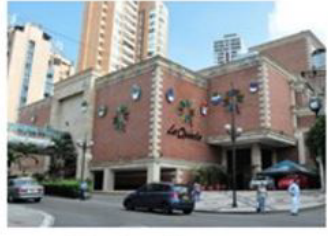

Contros Comerciales
Fuente: Coople mags

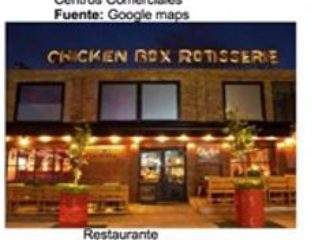

Ruentauranto: Coogle maps

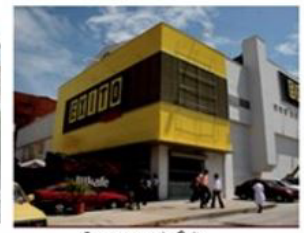

Supermercado Exilo
Fuente: Coople mags

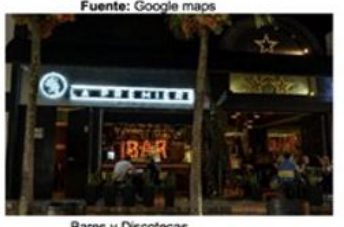

Bares y Discotocas
Fuente: Cocogie maps

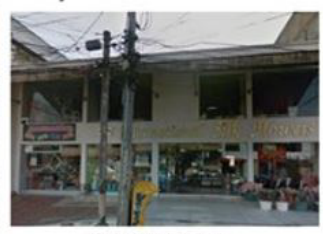

Comercio en general
Fuente: Googio maps

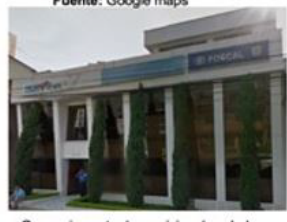

Comercio venta de servicios de salud
Fuente: Googioe maps

Figura 5: Uso del suelo comercial y servicios

Fuente: Elaboración propia a partir de Vera Rojas (2017).

Matriz de análisis urbano para el sector de estudio: Como parte de la discusión de resultados presentados en esta investigación y aporte como herramienta a los procesos pedagógicos para el estudio del ordenamiento y planificación territorial en la arquitectura se presenta el instrumento de matriz de análisis urbano, desarrollado al interior de esta investigación. Lo anterior bajo la consideración del establecimiento de un propósito general que definen aspectos significativos o de interés para la solución de problemáticas o fenómenos presentes en este tipo de estudios relacionados al territorio, asimismo con el fin de proponer orientaciones necesarias como respuesta a las tendencias o transformaciones del uso del suelo (Tabla 3 ). 
Tabla 3: Matriz de resultados MATRIZ DE RESULTADOS

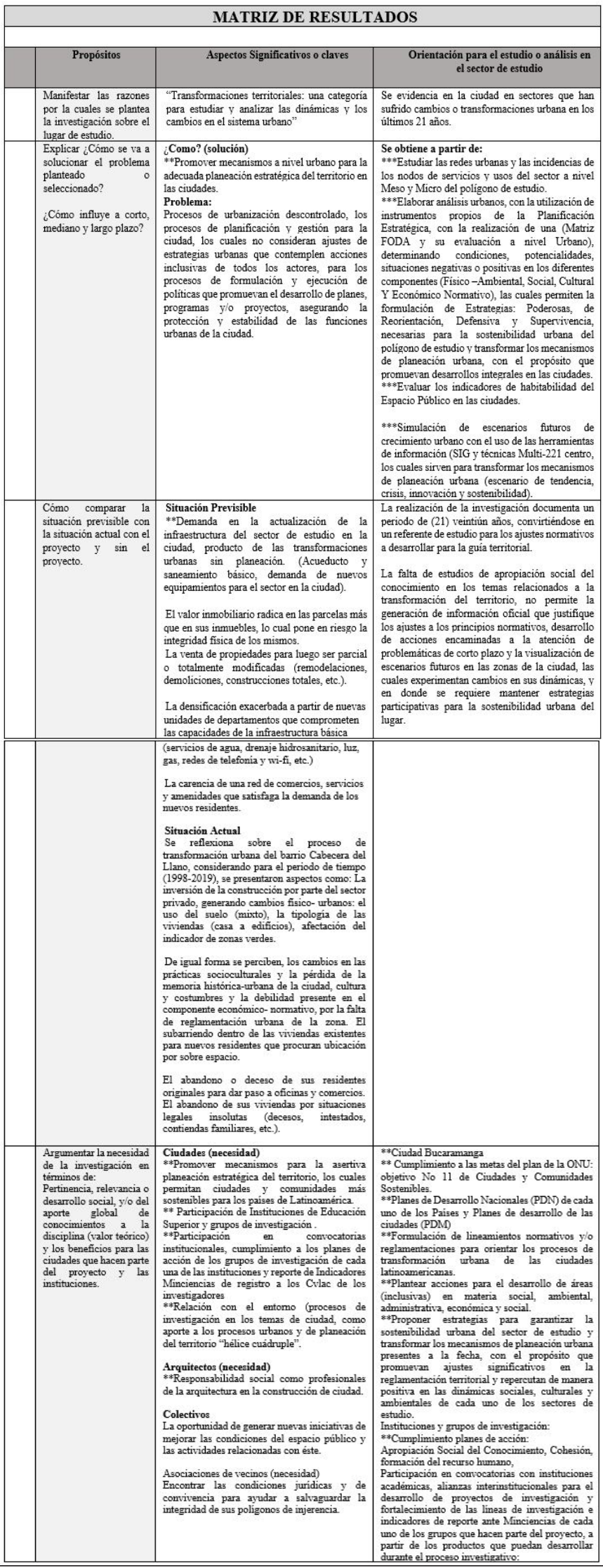




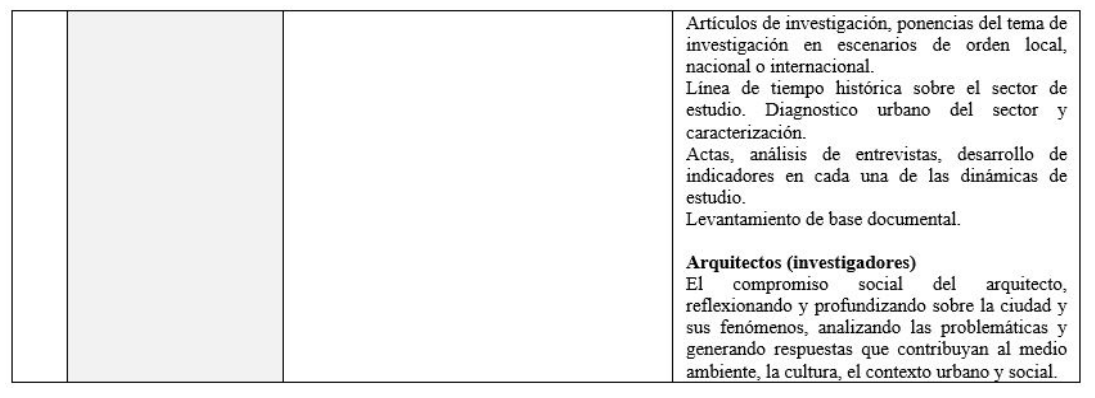

Fuente: Elaboración propia a partir Coronel Ruiz (2020)

\section{Conclusiones}

La presente investigación contribuyó en la generación de herramientas pedagógicas para el fortalecimiento del estudio y el entendimiento de las dinámicas urbanas, las transformaciones del territorio y los cambios en las tendencias de ocupación del suelo, a partir de la comprensión del ámbito histórico y el análisis territorial. De esta manera, se generó un estudio que permite consolidar elementos de interés a la gestión territorial como contribución al fortalecimiento del Plan de Ordenamiento Territorial y como generación de insumos para la toma de decisiones por parte de los entes gubernamentales pertinentes.

En materia de ordenamiento territorial, se determinó que el barrio Cabecera del Llano se vio influenciado notablemente con la aparición del uso del suelo comercial a gran escala (centros comerciales). A su vez se evidenciaron los cambios significativos en el ordenamiento territorial para el periodo de tiempo (1998-2019), a razón del crecimiento sin planeación estratégica y/o control urbano. Hecho que promovió problemáticas a nivel (físico-ambiental, social-cultural, económica y Normativa), cambios en la calidad de vida y el paisaje urbano, así como afectaciones en la habitabilidad y en los espacios públicos.

Por lo tanto, se establece la necesidad de gestar procesos que incrementen el control urbano referente al uso comercial del polígono de estudio. Esto, al tener en cuenta que dentro del rango de estudio (21 años) ha sido evidente el incremento de los locales comerciales, sin cumplimento a los aspectos normativos de la guía territorial, relacionado al manejo de antejardines y zonas de parqueo. Ante esta situación se evidencia, la necesidad de fortalecer la oficinas y procedimientos de control urbano, quienes deben ser los encargados de verificar el cumplimiento de las aprobaciones de licencias de construcción. Además, es importante generar estudios permanentes de carácter proyectivos, con relación a los cambios y tendencias de ocupación del uso del suelo teniendo en cuenta que las dinámicas allí presentes son altamente variables. Por lo tanto, las estrategias aplicadas deben ser flexibles, dinámicas y estar en constante evaluación por parte de los entes de control.

Con respecto al uso de los equipamientos, para el sector de Cabecera del Llano se recomienda aumentar el número de equipamientos dotacionales, ya que para el periodo de estudio el porcentaje de representación no es $100 \%$ significativo (cumplimiento básico mas no de proyección futura). Además, se recomienda analizar la capacidad de las redes y servicios de la zona a nivel urbano teniendo en cuenta la proyección de la población, para los siguientes años.

Igualmente, se sugiere hacer revisión normativa con respecto al uso residencial, teniendo en cuenta el incremento de los índices de construcción y las necesidades del mercado inmobiliario, considerando que esta zona aún se encuentra en una etapa de desarrollo y consolidación urbanística.

Con referencia a la influencia de esta problemática urbana a largo, mediano y corto plazo, 
se deben tener en cuenta los siguientes aspectos para la formulación de un plan de trabajo a nivel territorial:

Periodo largo plazo: La actualización de la infraestructura (Acueducto y saneamiento básico, demanda de nuevos equipamientos para el sector). La discontinuidad en el potencial de la identidad urbano-arquitectónica y socio-espacial del polígono de estudio.

Periodo mediano plazo: La desvalorización de los sectores residenciales, a partir de los cambios en los usos del suelo que se puedan presentar con alto impacto. Los cambios de la estructura socioespacial, condiciones de vida, representaciones sociales, y migraciones internas. La disminución del indicador de zonas verdes o especies arbóreas de los sectores de la ciudad, lo que lleva una afectación al medio ambiente.

Periodo corto plazo: La especulación en el mercado del suelo, por procesos de la actividad de construcción. La apuesta por el valor del suelo (adquisición-construcción -acondicionamientocomercialización y venta). El incremento de la actividad de licenciamiento y de construcción para la ciudad (repunte a los indicadores de construcción del municipio). La generación del fenómeno de burbuja inmobiliaria. La transformación de los perfiles urbanos. Los cambios de usos del suelo, promoviéndose el uso mixto, debido a la falta de control urbano y planeación y las alteraciones en el hábitat urbano.

\section{Referencias}

Aldunate, E. (2004). Metodología del Marco Lógico. Chile: Instituto Latinoamericano y

del Caribe de Planificación Económica y Social (ILPES).

Agencia de Ecología Urbana de Barcelona. (2008). Plan especial de indicadores de sostenibilidad ambiental de la actividad urbanística de
Sevilla. Barcelona: Servicio de observatorio de Sostenibilidad Urbanística.

Banco Interamericano de Desarrollo- BID (1997). Anexo I. La Matriz de Marco Lógico,

en Evaluación: una herramienta de gestión para mejorar el desempeño de los

proyectos. Washington: BID

Bernal Granados, M. A. (2015). Análisis y diagnóstico urbano regional metodología para la caracterización territorial. Bogotá: Universidad Piloto de Colombia.

Beuf, A. (2012). Concepción de centralidades urbanas y planeación del crecimiento urbano en la Bogotá del siglo XX. XII coloquio internacional de Geocritica, 1-21.

Bryman, A. (1998). Quantity and Quality in social research. Londres: Unwin Hyman Carr.

Coronel Ruiz, L. K (2020) Matriz de consideración de justificación en procesos de análisis urbanoterritorial. Universidad Francisco de Paula Santander.

CEPAL (2013). Guía análisis del sistema urbano regional para el ordenamiento territorial, División de Desarrollo Sostenible y Asentamientos Humanos Comisión Económica para América Latina y el Caribe, Chile.

Concejo de Bucaramanga (2000). Plan de Ordenamiento territorial municipio de Bucaramanga año 2000. Acuerdo No 034 del 25 de septiembre 2000 .

Concejo de Bucaramanga (2014). Plan de Ordenamiento territorial segunda generación del municipio de Bucaramanga 2014-2027. Acuerdo 011 de mayo 2014.

Contreras, Y. (2009). Movilidad residencial centrípeta: el rol del mercado inmobiliario y del 
nuevo habitante urbano en la recuperación del centro de Santiago de Chile. XII Encuentro de Geógrafos de América Latina "Caminando en una América Latina en transformación" Chile.

Estévez Suarez, F. A. (2018). Transformación del territorio por dinámicas urbanas en el sector de Cañaveral municipio de Floridablanca. Bucaramanga: Universidad Santo Tomas.

Gallardo Frías, L. (2015). Metodología de análisis del contexto. Aproximación interdisciplinar. VII Seminario Internacional de Investigación en Urbanismo, Chile.

Gill, J., \& Johnson, P. (2010). Research methods for managers. Londres: Sage Publications.

Guash, O. (1997). Observación participante. Madrid: Centro de investigaciones metodológicas.

Martínez R, L. (2007). La observación y el diario de campo en la definición de un tema de investigación. Perfiles Libertadores. Fundación Universitaria los libertadores, 73-80.

Molina Azorín, J. (2012). Mixed methods research in strategics management impacts and applications. Organizational Research Methods, 15(1), 33-56.

Rueda Gómez, N. J. (2012). La formación del Área Metropolitana de Bucaramanga: el papel de la vivienda del Instituto de Crédito Territorial como elemento clave de su configuración. Bucaramanga.

Rueda Gómez, N. J. (2013). Paradojas de un ordenamiento urbano. Bucaramanga: Universidad Santo Tomas.

Skinner, D., Hollovay, J., \& Tagg, C. (2000). Managers and researh: The prosand cons of qualitative approaches. Sage Journals. la vida. Fundamentos y metodologías para la investigación educativa social. Revista Fundación Universitaria Luis Amigó.

Vera Rojas, S. L (2017). Nuevas Centralidades en la Ciudad de Bucaramanga: Caso Cabecera del Llano. Tesis de pregrado. Universidad Santo Tomas.

Vera, S. (2018) Nuevas Centralidades en la Ciudad de Bucaramanga: Caso Cabecera del Llano tesis de pregrado.

World Bank (S.F) The logframe handbook. A logical framework approach to project cycle management

Vélez de la Calle, C. (1999). Las historias de 